\title{
Caracterización de vermicompostas y su efecto en la germinación y crecimiento de Capsicum chinense Jacquin
}

\section{Characterization of vermicomposts and their effect on the germination and growth of Capsicum chinense Jacquin}

\author{
Jesse Joel Edson David-Santoya ${ }^{1}$, Regino Gómez-Álvarez ${ }^{1 *}$, Aarón Jarquín-Sánchez ${ }^{1}$, Gilberto \\ Villanueva-López ${ }^{1}$ \\ ${ }^{1}$ El Colegio de la Frontera Sur, Carretera a Reforma Km 15.5 s/n, Ra. Guineo Segunda Sección. CP. 86280. Centro, Tabasco, \\ México. \\ *Autor de correspondencia: regomez@ecosur.mx
}

Artículo científico recibido: 16 de mayo de 2017 aceptado: 13 de septiembre de 2017

RESUMEN. El uso de diferentes sustratos para la elaboración de vermicompostas tienen influencia en el desarrollo de los cultivos. El objetivo fue evaluar la productividad de la lombriz Eisenia andrei en diferentes sustratos y el efecto en la germinación y crecimiento del Capsicum chinense Jacquin. Se elaboraron vermicompostas con residuos vegetales (RV) y cascarilla de cacao (CC), y sus combinaciones con Gliricidia sepium (G). Los contenidos de ácidos húmicos (AH) y carbono de ácidos húmicos (CAH) tuvieron los mayores valores en las vermicompostas con RV, estiercol de ovino (EO) y sus combinaciones con G. El EO incrementó la biomasa y longitud de la lombriz, al combinarlo con $\mathrm{G}$ incremento la mortalidad de cocones y lombrices juveniles. Con el bocashí (B) y los RV se tuvieron efectos positivos en la biomasa, longitud, número de cocones y lombrices juveniles. Las vermicompostas de $B, B+G+R V$ y $R V$ tuvieron los mayores porcentajes de germinación. Para incrementar la biomasa de la lombriz, la población, la longitud, el número de cocones y el número de lombrices juveniles se pueden usar sustratos elaborados con RV, B y sus combinaciones. Los mayores contenidos de nutrientes en las plántulas de Capsicum chinense Jacquin se obtuvieron en la combinación de EO+G.

Palabras clave: Abonos orgánicos, chile habanero, desechos orgánicos, Eisenia andrei, sustratos

ABSTRACT. The use of different substrates for producing vermicomposts has an impact on crop development. The aim was to evaluate the productivity of the Eisenia andrei earthworm in different substrates and their effect on the germination and growth of Capsicum chinense Jacquin. They were prepared with plant residues (PR) and cocoa husk $(\mathrm{CH})$, and their combinations with Gliricidia sepium $(\mathrm{G})$. The contents of humic acids (HA) and carbon of humic acids (CAH) had the highest values in the vermicomposts with $\mathrm{PR}$, sheep manure (SM) and their combinations with $G$. The SM increased the biomass and length of the earthworm, whereas combining it with $G$ increased the mortality of cocoons and juvenile worms. With bocashi (B) and PR there were positive effects on the biomass, length, number of cocoons and juvenile worms. The vermicomposts of $B, B+G+P R$ and $P R$ had the highest germination percentages. To increase earthworm biomass, population, length, number of cocoons and the number of juvenile worms, substrates made with PR, B and their combinations can be used. The highest nutrient contents in Capsicum chinence Jacquin seedlings were found in the $\mathrm{SM}+\mathrm{G}$ combination.

Key words: Organic fertilizers, habanero pepper, organic waste, Eisenia andrei, substrates

\section{INTRODUCCIÓN}

El vermicompostaje es un método que se usa para el manejo de residuos orgánicos, con la ayuda de lombrices de tierra y microorganismos (Bhat et al. 2015). Las lombrices rompen y digieren los desechos orgánicos y los transforman en vermicompostas (Atiyeh et al. 2002). Las excelentes propiedades físicas, químicas y biológicas convierten a las vermicompostas en excelentes fertilizantes orgánicos que 


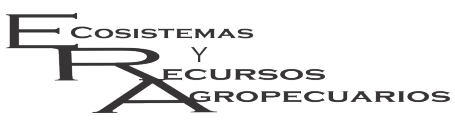

se usan como sustratos para las plantas (McGinnis et al. 2009); las propiedades de las vermicompostas dependen de los residuos utilizados (Atiyeh et al. 2002). Al respecto Moreno-Reséndez et al. (2005), Rodríguez et al. (2007) y (2008) reportan que las vermicompostas tienen una gran variedad en la composición y calidad de las sustancias húmicas que contienen.

La estabilidad de las vermicompostas se relaciona con la madurez del abono (Bazrafshan et al. 2016). Por ejemplo, vermicompostas inmaduras inhiben el crecimiento de las plantas, debido a que producen sustancias fitotóxicas que causan deficiencias de nitrógeno, lo que ocasiona competencia en las raíces y los microorganismos edáficos por compuestos nitrogenados (Majlessi et al. 2012). Por lo que vermicompostas maduras mejoran las propiedades físicas, químicas, biológicas y la estructura del suelo, lo que incrementa el rendimiento de los cultivos, y aumenta la disponibilidad de nutrientes, la población microbiana y los metabolitos (Atiyeh et al. 2002). Con respecto a la aplicación de vermicompostas en el cultivo de chile, NietoGaribay et al. (2002) encontraron que la mejor dosis de vermicomposta en campo fue de $25 \mathrm{t} \mathrm{ha}^{-1}$; mientras que Barreto et al. (2008) reportan que aplicar vermicomposta a razón de $100 \mathrm{~g}$ por kg de suelo, mejora el $\mathrm{pH}$ y la materia orgánica del suelo, lo que impacta en mayor rendimiento y altura de planta. En tanto que Tamayo et al. (2014) y Ruíz-Bello et al. (2016) obtuvieron mejoras en el suelo e incrementos en los rendimientos al aplicar vermicompostas en plántulas y campo. Por lo anterior el objetivo del presente estudio fue evaluar la productividad de la lombriz Eisenia andrei en diferentes sustratos, y el efecto en la germinación y crecimiento de Capsicum chinense Jacquin.

\section{MATERIALES Y MÉTODOS}

\section{Área de estudio}

El experimento se realizó en el campo experimental de El Colegio de la Frontera Sur (ECOSUR) Unidad Villahermosa, ubicado en la Ranchería Guineo Segunda Sección del municipio de Centro,
Tabasco. Se localiza entre los $17^{\circ} 54^{\prime} \mathrm{LN}$ y $93^{\circ}$ 02 ' LO, a una altitud de $12 \mathrm{msnm}$; la temperatura promedio anual es de $27.2{ }^{\circ} \mathrm{C}$, humedad relativa del $84 \%$ y precipitación de $1749.7 \mathrm{~mm}$ al año.

\section{Materiales utilizados}

El experimento se realizó de marzo a diciembre de 2016. Se evaluó la producción de vermicompostas con estiércol de ovino (EO), residuos vegetales (RV), bocashí (B), hojas y tallos de $G$. sepium $(G)$, cascarilla de cacao (CC) y sus combinaciones, bajo diseño experimental de bloques completos al azar con cuatro repeticiones (Tabla 1). El EO provino del Centro de Capacitación y Reproducción de Especies Menores (CECAREM) del municipio de Centro, Tabasco. La hojarasca fresca y seca se recolectó en el campo experimental, mientras que la G. sepium (G) y la cascarilla de cacao (CC) se colectaron en plantaciones cercanas a la Unidad Villahermosa de ECOSUR.

\section{Elaboración del bocashí}

Para elaborar el bocashi se colocaron en capas el suelo, estiércol, cenizas, cal industrial, melaza, aserrín, levadura, carbón y agua. Posteriormente se mezclaron, humedecieron y se cubrieron con polietileno. La fermentación se realizó por dos semanas, en la primera semana se mezcló dos veces al día, y en la segunda se realizó la mezcla cada dos días (Ramos y Terry 2014).

\section{Precomposteo de sustratos orgánicos}

El precomposteo se realizó por ocho semanas, para lo cual se picó, mezcló, humedeció a capacidad de campo, tapó y mantuvo la humedad al $70 \%$ de los diferentes sustratos orgánicos, hasta que la mezcla llegó a temperatura ambiente. Para la transformación de los materiales orgánicos precomposteados en vermicompostas se utilizó la lombriz Eisenia andrei (Bouché 1972), obtenida de la Unidad experimental de Lombricultura de ECOSUR. Se introdujeron 50 lombrices con longitud promedio de $3.0 \mathrm{~cm}$ y peso de $0.12 \mathrm{~g}$ en macetas de plástico, a las que se agregaron $2.8 \mathrm{~kg}$ del sustrato precomposteado de los diferentes tratamientos. Para mantener la 
Tabla 1. Sustratos utilizados para vermicompostaje con lombriz Eisenia andrei.

\begin{tabular}{lc}
\hline Composición & Porcentaje \\
\hline Estiércol ovino (EO) & $100 \%$ \\
Estiércol ovino + G. sepium (EO+G) & $50 \%+50 \%$ \\
Bocashí (B) & $100 \%$ \\
Bocashí + G. sepium + residuos vegetales (B+G+RV) & $50 \%+25 \%+25 \%$ \\
Residuos vegetales (RV) & $100 \%$ \\
Residuos vegetales + G. sepium (RV+G.) & $50 \%+50 \%$ \\
Cascarilla de cacao (CC) & $100 \%$ \\
Cascarilla de cacao + G. sepium (CC+G.) & $50 \%+50 \%$ \\
Cascarilla de cacao + G. sepium + bocashí (CC+G+B) & $50 \%+25 \%+25 \%$ \\
Cascarilla de cacao + G. sepium + residuos vegetales (CC+G+RV) & $50 \%+25 \%+25 \%$ \\
Cascarilla de cacao + G. sepium + estiércol ovino (CC+G+EO) & $50 \%+25 \%+25 \%$ \\
G. sepium (G) & $100 \%$ \\
\hline
\end{tabular}

humedad al $70 \%$ se realizaron riegos continuos de acuerdo con Batham et al. (2014). Las macetas se tuvieron bajo malla sombra al $50 \%$ de luminosidad por cuatro semanas, para luego recolectar, contar y medir las lombrices, para evaluar el porcentaje de mortalidad, biomasa, longitud promedio de las lombrices adultas y juveniles, y contabilizar el número de cocones. Para realizar la medición y conteo de las lombrices, se tomaron $500 \mathrm{~g}$ de las vermicompostas, para luego secar a temperatura ambiente y realizar los análisis químicos.

\section{Análisis químico}

Los análisis químicos del suelo y las vermicompostas se realizaron en el laboratorio de Biogeoquímica de ECOSUR. El pH se determinó por el método AS-02 en un extracto vermicomposta-suelo, en relación 1:5; el nitrógeno total por el método AS-25; mientras que la capacidad de intercambio catiónico $(\mathrm{CIC})$ y los cationes intercambiables $\left(\mathrm{Ca}^{+2} \mathrm{y} \mathrm{K}^{+}\right)$se determinó con el método AS-12 (SEMARNAT 2000). El contenido de fósforo total se determinó con el método colorimétrico de azul de molibdeno. Los ácidos húmicos por extracción alcalina con pirofosfato de sodio. La materia orgánica (MO) y el carbono orgánico total (COT) se determinaron por el método de calcinación (Nelson y Sommers 1996). La relación C/N se calculó con los valores iniciales del carbono de la materia orgánica y el nitrógeno total.

\section{Bioensayo}

Para determinar la calidad de las vermicom- postas se realizó un experimento de germinación de semillas de chile habanero (Capsicum chinense Jacquin) variedad Mayapán. Las vermicompostas se mezclaron con suelo en proporción (1:1). El suelo (Tabla 2) utilizado fue un Fluvisol eútrico (PalmaLópez et al. 2007), que se colectó en ECOSUR. Las mezclas de los sustratos se colocaron en bandejas de germinación con 80 cavidades, para luego sembrar una semilla por cavidad. Para evaluar la germinación de las semillas se utilizó un diseño de bloques completos al azar con tres repeticiones por tratamiento. Las charolas se regaron todos los días para asegurar que la humedad no fuera un factor limitante. Las variables evaluadas fueron la germinación de las semillas a los 21. Para evaluar la nutrición de las plantas a los $60 \mathrm{~d}$ después de la siembra se determinó la biomasa fresca por gravimetría, se contabilizó el número de hojas, midió la altura de planta, la longitud de raíz y el área foliar. También se seleccionaron de forma aleatoria 10 plantas por repeticiones, para realizar los análisis de $\mathrm{N}, \mathrm{P}$ y K ; el contenido de $\mathrm{N}$ se determinó con el método Semimicro Kjeldahl, mientras que el contenido total de $\mathrm{P}$ y $\mathrm{K}$ se determinó por colorimetría y espectrofotometría de llama (SEMARNAT 2000).

\section{Análisis estadístico}

Los datos se analizaron con el Paquete Estadístico R Studio Versión 0.98.1103. Los análisis de crecimiento de las lombrices, características químicas de las vermicompostas y las variables de crecimiento de las plantas se realizaron mediante un análisis de varianza (ANOVA). Para determinar 


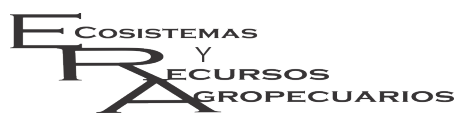

diferencias entre medias se realizó la comparación de medias de Tukey $(p \leq 0.05)$.

Tabla 2. Caracterización físico-química del suelo (Fluvisol eútrico).

\begin{tabular}{lc}
\hline Característica & Contenido \\
\hline $\mathrm{pH}$ & 6.7 \\
Arena (\%) & 10.0 \\
Limo (\%) & 48.0 \\
Arcilla (\%) & 42.0 \\
Clasificación & Arcillo limoso \\
Materia orgánica (\%) & 8.4 \\
ClC (cmol kg $\left.{ }^{-1}\right)$ & 12.2 \\
Nitrógeno Total $(\%)$ & 0.14 \\
Fósforo Total $(\%)$ & 0.024 \\
Potasio (cmol kg ${ }^{-1}$ ) & 0.85 \\
\hline CIC: Capacidad de & intercambio \\
catiónico. &
\end{tabular}

\section{RESULTADOS}

\section{Caracterización de la lombriz Eisenia andrei en} los diferentes sustratos orgánicos

La sobrevivencia de las lombrices en los diferentes sustratos precomposteados se evaluó a las 24 h después de la inoculación, debido a que es el tiempo para que se estabilice el sistema (Tabla 3). El mayor porcentaje de mortalidad de lombrices se observó en los tratamientos con 50\% EO+50\% $\mathrm{G}$ con el $27 \%$ de lombrices muertas, el tratamiento $50 \%$ RV $+50 \%$ G con el $20.5 \%$ y el tratamiento $100 \%$ EO con $8 \%$. No se encontraron lombrices muertas en los tratamientos $100 \%$ CC, $50 \%$ CC $+25 \%$ G $+25 \%$ B y $50 \%$ CC $+25 \%$ G $+25 \%$ RV. Después de los $60 \mathrm{~d}$ de vermicomposteo, se observaron incrementos poblacionales en todos los tratamientos, con excepción del tratamiento con $50 \% \mathrm{EO}+50 \% \mathrm{G}$, donde no se encontraron lombrices juveniles. La máxima población se observó en el tratamiento con $100 \%$ B con 389 lombrices. Para la producción de cocones se encontraron diferencias significativas $(p \leq 0.05)$ entre los tratamientos, la mayor producción se encontró en $100 \%$ RV con 422 cocones, y la mínima producción en el tratamiento $100 \%$ EO con 16 . No se contabilizaron cocones en el tratamiento con $50 \% \mathrm{EO}+50 \% \mathrm{G}$, en tanto que las mayores cantidades de lombrices juveniles se tuvieron en el tratamiento con $100 \%$ B con 342 , mientras que el menor número de lombrices juveniles
Efecto de vermicompostas en Capsicum chinense

Ecosist. Recur. Agropec.

5(14):181-190,2018

se tuvo en los tratamientos $100 \%$ EO, y $50 \%$ EO $+50 \%$ G. Se encontraron diferencias significativas $(p \leq 0.05)$ en la biomasa de lombrices adultas en los tratamientos, presentando la mayor biomasa el tratamiento con $50 \%$ EO $+50 \%$ G con $0.43 \mathrm{~g}$. Para la longitud de las lombrices se encontraron diferencias significativas $(p \leq 0.05)$ entre tratamientos, observándose que las mayores longitudes de lombrices estuvieron en los tratamientos 50\% EO + $50 \%$ G con $5.7 \mathrm{~cm}$, y $100 \%$ EO con $5.1 \mathrm{~cm}$, mientras que las menores longitudes se tuvieron en los tratamientos con CC (Tabla 3).

\section{Caracterización de las vermicompostas}

En la Tabla 4 se observan las características químicas de las diferentes vermicompostas. Se tuvieron diferencias significativas $(\mathrm{p} \leq 0.05)$ en el $\mathrm{pH}$, con valores entre 7.9 y 8.1 en los tratamientos con $50 \%$ RV + 50\% G, $100 \%$ CC, $50 \%$ CC + 25\% $\mathrm{G}+25 \% \mathrm{~B}$, y $50 \% \mathrm{CC}+25 \% \mathrm{G}+25 \% \mathrm{RV}$, valores aceptables para sustratos orgánicos. Para el contenido de MO (72 a $82 \%), C$ (42 a $47 \%$ ) y N (1.4 a 1.6\%) se tuvieron los mayores valores en los tratamientos con residuos vegetales y cascarilla de cacao, y cuando se combinaron con G. sepium (RV, $\mathrm{RV}+\mathrm{G}, \mathrm{CC}$ y $\mathrm{CC}+\mathrm{G})$.

La cascarilla de cacao disminuyó los contenidos de carbono en los ácidos húmicos (4.3 a 5.3\%), lo que influyó de forma positiva en la relación C/N (24 a 38\%) (Tabla 4). La mayoría de los tratamientos tuvieron una relación $\mathrm{C} / \mathrm{N}$ entre 20 y 30 , lo que significa una lenta tasa de mineralización, por lo que no existieron pérdidas significativas de carbono durante el proceso de vermicomposteo. La mayor capacidad de intercambio catiónico a los $60 \mathrm{~d}$ de vermicompostaje se encontró en los sustratos, $C C$ y $C C+G$ y los menores en $B$ y $B+G+R V$. Para el contenido de potasio los mayores valores lo tuvieron los tratamientos con cascarilla de cacao con valores entre 61.4 y 86.3

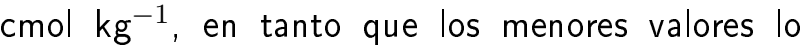
tuvieron los tratamientos con residuos vegetales. Para el contenido de calcio el mayor valor lo tuvo el tratamiento $\mathrm{CC}+\mathrm{G}+\mathrm{EO}$ con $33.7 \mathrm{cmol} \mathrm{kg}^{-1} \mathrm{y}$ los menores valores los tratamientos CC $(13.7 \mathrm{cmol}$ 
Tabla 3. Efecto de los diferentes sustratos en la reproducción de la lombriz Eisenia andrei.

\begin{tabular}{cccccccc}
\hline Composición & Relación & Biomasa $(\mathrm{g})$ & $\begin{array}{c}\text { Número de } \\
\text { lombrices }\end{array}$ & $\begin{array}{c}\text { Longitud } \\
(\mathrm{cm})\end{array}$ & $\begin{array}{c}\text { Número de } \\
\text { Cocones }\end{array}$ & $\begin{array}{c}\text { Número de } \\
\text { Juveniles }\end{array}$ & $\begin{array}{c}\text { Mortalidad } \\
(24 \mathrm{~h})(\%)\end{array}$ \\
\hline EO & 1 & $0.28^{c d}$ & $86^{e}$ & $5.1^{a b}$ & $16^{f g}$ & $41^{e f}$ & 8.0 \\
EO+G & $1: 1$ & $0.43^{a}$ & $37^{f}$ & $5.7^{a}$ & 0 & 0 & 27.0 \\
B & 1 & $0.26^{d}$ & $389^{a}$ & $4.7^{b}$ & $138^{c d}$ & $342^{a}$ & 6.5 \\
B+G+RV & $2: 1: 1$ & $0.29^{c d}$ & $240^{b}$ & $4.6^{b}$ & $162^{c}$ & $110^{c d}$ & 4.5 \\
RV & 1 & $0.37^{a b}$ & $249^{b}$ & $5.2^{a b}$ & $422^{a}$ & $206^{b}$ & 4.5 \\
RV+G & $1: 1$ & $0.33^{b c}$ & $182^{c}$ & $4.7^{b}$ & $220^{b}$ & $113^{c d}$ & 20.5 \\
CC & 1 & $0.16^{e}$ & $113^{d e}$ & $3.2^{c}$ & $129^{d e}$ & $63^{e}$ & 0 \\
CC+G & $1: 1$ & $0.15^{e}$ & $163^{c}$ & $3.2^{c}$ & $153^{c d}$ & $114^{c d}$ & 1.5 \\
CC+G+B & $2: 1: 1$ & $0.17^{e}$ & $177^{c}$ & $3.5^{c}$ & $147^{c d}$ & $127^{c}$ & 0 \\
CC+G+RV & $2: 1: 1$ & $0.17^{e}$ & $123^{d}$ & $3.5^{c}$ & $103^{e}$ & $73^{d e}$ & 0 \\
CC+G+EO & $2: 1: 1$ & $0.17^{e}$ & $96^{d e}$ & $3.5^{c}$ & $40^{f}$ & $48^{e}$ & 4.0 \\
Media & - & 0.25 & 168.4 & 4.3 & 139 & 112 & 7.6 \\
CV\% & - & 11.5 & 6.5 & 7.4 & 9.4 & 16.9 & -
\end{tabular}

Medias con letras diferentes difieren de forma significativa (Tukey, $\mathrm{p} \leq 0.05$ ).

Tabla 4. Caracterización química de las vermicompostas.

\begin{tabular}{|c|c|c|c|c|c|c|c|c|c|c|c|}
\hline Composición & $C / N$ & $\mathrm{pH}$ & MO & C & $\%^{\mathrm{N}}$ & $\mathrm{AH}$ & $\mathrm{CAH}$ & $\mathrm{P}$ & K & $\begin{array}{l}\mathrm{Ca} \\
\mathrm{cmol} / \mathrm{kg}\end{array}$ & $\mathrm{CIC}$ \\
\hline $\mathrm{EO}$ & $18.0^{c}$ & $8.1^{a b}$ & $47.0^{g}$ & $28.0^{g}$ & $1.5^{b c}$ & $0.3^{a}$ & $8.8^{b c}$ & $1.4^{a}$ & $20.4^{d}$ & $25.0^{b c d}$ & $60.8^{d}$ \\
\hline $\mathrm{EO}+\mathrm{G}$ & $23.0^{b c}$ & $8.0^{a b}$ & $53.0^{f}$ & $31.0^{f}$ & $1.4^{b c}$ & $0.2^{a b c}$ & $11.2^{a b}$ & $1.3^{a}$ & $45.4^{c}$ & $27.5^{a b c}$ & $63.1^{d}$ \\
\hline B & $26.0^{b}$ & $8.0^{a b c d}$ & $36.0^{i}$ & $21.0^{i}$ & $0.8^{e}$ & $0.2^{b c}$ & $8.7^{b c}$ & $0.6^{c}$ & $16.8^{d}$ & $20.0^{\text {cde }}$ & $44.3^{e}$ \\
\hline$B+G+R V$ & $25.0^{b}$ & $7.8^{b c d}$ & $43.0^{h}$ & $25.0^{h}$ & $1.0^{d e}$ & $0.3^{a b c}$ & $6.7^{b c}$ & $0.6^{c}$ & $16.2^{d}$ & $20.6^{\text {cde }}$ & $52.2^{e}$ \\
\hline RV & $28.0^{b}$ & $7.8^{a b c d}$ & $73.0^{c}$ & $42.0^{c}$ & $1.5^{b c}$ & $0.3^{a b c}$ & $11.8^{a b}$ & $0.4^{d e f}$ & $11.9^{d}$ & $30.6^{a b}$ & $77.0^{c}$ \\
\hline $\mathrm{RV}+\mathrm{G}$ & $25.0^{b}$ & $8.0^{a b c}$ & $72.0^{c}$ & $42.0^{c}$ & $1.6^{a b}$ & $0.2^{c}$ & $14.1^{a}$ & $0.5^{c d}$ & $22.3^{d}$ & $29.3^{a b}$ & $71.0^{c}$ \\
\hline $\mathrm{CC}$ & $38.0^{a}$ & $7.9^{a b c d}$ & $82.0^{a}$ & $47.0^{a}$ & $1.3^{c d}$ & $0.3^{a b c}$ & $4.6^{c}$ & $0.2^{g}$ & $66.5^{b}$ & $13.7^{e}$ & $101.4^{a}$ \\
\hline $\mathrm{CC}+\mathrm{G}$ & $24.0^{b}$ & $7.7^{d}$ & $77.0^{b}$ & $45.0^{b}$ & $1.8^{a}$ & $0.3^{a b c}$ & $5.1^{c}$ & $0.3^{e f g}$ & $86.3^{a}$ & $16.8^{e}$ & $101.5^{a}$ \\
\hline $\mathrm{CC}+\mathrm{G}+\mathrm{B}$ & $28.0^{b}$ & $7.9^{a b c d}$ & $61.0^{e}$ & $35.0^{e}$ & $1.3^{c d}$ & $0.3^{a b c}$ & $5.2^{c}$ & $0.4^{d e}$ & $61.4^{b}$ & $13.7^{e}$ & $71.0^{c}$ \\
\hline$C C+G+R V$ & $24.0^{b}$ & $8.1^{a}$ & $64.0^{d}$ & $37.0^{d}$ & $1.5^{b c}$ & $0.3^{a b c}$ & $5.3^{c}$ & $1.1^{b}$ & $62.7^{b}$ & $19.3^{d e}$ & $71.2^{c}$ \\
\hline $\mathrm{CC}+\mathrm{G}+\mathrm{EO}$ & $29.0^{b}$ & $7.8^{c d}$ & $80.0^{a}$ & $47.0^{a}$ & $1.6^{a b}$ & $0.3^{a b}$ & $5.2^{c}$ & $0.2^{f g}$ & $71.6^{b}$ & $33.7^{a}$ & $91.0^{b}$ \\
\hline Media & 26.4 & 7.9 & 62.6 & 36.3 & 1.4 & 0.25 & 7.9 & 0.6 & 43.8 & 22.8 & 73.1 \\
\hline CV \% & 9.0 & 1.4 & 1.3 & 1.3 & 8.9 & 16.2 & 26.8 & 10.2 & 13.0 & 14.3 & 4.3 \\
\hline
\end{tabular}

Medias con letras diferentes difieren significativamente entre si según test de Tukey ( $\mathrm{p} \leq 0.05)$, $\mathrm{AH}$ : ácidos húmicos y $\mathrm{CAH}$ : carbono de ácidos húmicos.

$\left.\mathrm{kg}^{-1}\right), \mathrm{C}+\mathrm{G}+\mathrm{B}\left(13.7 \mathrm{cmol} \mathrm{kg}^{-1}\right)$ y $\mathrm{CC}+\mathrm{G}(16.8$

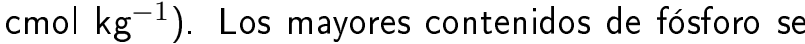
encontraron en los tratamientos con $\mathrm{EO}$ y $\mathrm{EO}+\mathrm{G}$ (1.4 y $1.3 \%$ ), y los menores los tratamientos con CC.

\section{Bioensayo vegetal}

La germinación de semillas de Capsicum chinense se presenta en la Tabla 5. A los $21 \mathrm{~d}$, se tuvo el mayor porcentaje de germinación en los tratamientos B + Suelo (41\%), RV + Suelo (38\%) y el testigo (33\%). Las plántulas del tratamiento testigo tuvieron bajo crecimiento, lo que confirma que este sustrato no suministró los nutrientes necesario para el desarrollo de las plántulas. En los tratamientos $E O$ y $E O+G$ se tuvieron bajos porcentajes de germinación y desarrollo de las plántulas de chile, lo que se puede deber a la baja degradación del estiércol ovino y la $G$. sepium, pero también se tuvo en este tratamiento altos contenidos de $\mathrm{N}$, lo que pudo influir en la germinación, crecimiento y desarrollo de las plántulas de chile.

Altura de planta, área foliar y número de hojas La combinación de vermicomposta y suelo $(1: 1)$ incrementó de forma significativa $(\mathrm{p} \leq 0.05)$ la altura y el área foliar de las plántulas de chile habanero al compararse con el testigo. La mayor altura y área foliar se encontró en los tratamientos con B + Suelo $(8.4 \mathrm{~cm})$ y $C C+G+E O+$ Suelo $\left(3.0 \mathrm{~cm}^{2}\right)$, respectivamente. Se detectaron diferencias significativas $(\mathrm{p} \leq 0.05)$ para la longitud de raíz, observándose la mayor longitud en B + Suelo (8.8 
Tabla 5. Indicadores de crecimiento y contenidos de nutrientes en plántulas de chile habanero.

\begin{tabular}{|c|c|c|c|c|c|c|c|c|c|}
\hline \multirow[b]{2}{*}{ Sustratos } & \multicolumn{5}{|c|}{ Indicadores de crecimiento } & \multicolumn{3}{|c|}{ Contenido de nutrientes } & \multirow{2}{*}{$\begin{array}{c}\text { Germinación } \\
(\%)\end{array}$} \\
\hline & $\begin{array}{l}\text { Area } \\
\text { foliar } \\
\left(\mathrm{cm}^{2}\right)\end{array}$ & $\begin{array}{c}\text { Altura } \\
\text { de Planta } \\
\left(\mathrm{cm}^{2}\right)\end{array}$ & $\begin{array}{c}\text { Longitud } \\
\text { de Raíz } \\
(\mathrm{cm})\end{array}$ & $\begin{array}{l}\text { Número } \\
\text { de hojas }\end{array}$ & $\begin{array}{c}\text { Biomasa } \\
\text { Fresca } \\
(\mathrm{g})\end{array}$ & $\mathrm{N}$ & $\begin{array}{l}P \\
(\%)\end{array}$ & K & \\
\hline Suelo & $2.3^{a b}$ & $8.4^{a}$ & $8.8^{a}$ & $7.6^{b c}$ & $0.4^{a b}$ & $3.4^{a b}$ & $0.2^{a b}$ & $0.55^{a b c}$ & \\
\hline$B+G+R V+$ Suelo & $2.3^{a b}$ & $7.1^{b c}$ & $6.0^{b c}$ & $8.3^{a b}$ & $0.4^{a b c}$ & $3.9^{a}$ & $0.1^{d}$ & $0.59^{a b c}$ & 32.4 \\
\hline RV+Suelo & $1.6^{b c}$ & $4.7^{d}$ & $5.0^{c}$ & $6.4^{d}$ & $0.2^{c d}$ & $3.0^{a b c}$ & $0.2^{b c}$ & $0.65^{a b c}$ & 38.7 \\
\hline $\mathrm{RV}+\mathrm{G}+$ Suelo & $1.6^{b c}$ & $5.4^{d e}$ & $5.4^{c}$ & $7.1^{c d}$ & $0.2^{c d}$ & $3.2^{a b c}$ & $0.1^{c d}$ & $0.53^{b c}$ & 22.5 \\
\hline $\mathrm{CC}+\mathrm{G}+$ Suelo & $2.2^{a b}$ & $6.8^{e}$ & $6.3^{b c}$ & $8.0^{a b c}$ & $0.3^{b c d}$ & $3.2^{a b c}$ & $0.2^{a b}$ & $0.74^{a}$ & 23.3 \\
\hline $\mathrm{CC}+\mathrm{G}+\mathrm{B}+$ Suelo & $2.1^{a b}$ & $6.3^{c d}$ & $5.0^{c}$ & $8.1^{a b c}$ & $0.4^{a b c}$ & $2.8^{b c}$ & $0.2^{a b}$ & $0.68^{a b c}$ & 32.2 \\
\hline $\mathrm{CC}+\mathrm{G}+\mathrm{RV}+$ Suelo & $2.3^{a b}$ & $7.3^{a b c}$ & $5.8^{c}$ & $8.2^{a b}$ & $0.4^{a b}$ & $3.2^{a b c}$ & $0.3^{a}$ & $0.63^{a b c}$ & 33.8 \\
\hline $\mathrm{CC}+\mathrm{G}+\mathrm{EO}+$ Suelo & $3.0^{a}$ & $8.1^{a b}$ & $7.9^{a b}$ & $8.7^{a}$ & $0.5^{a}$ & $3.2^{a b c}$ & $0.2^{a b}$ & $0.72^{a b}$ & 22.9 \\
\hline Suelo (Testigo) & $0.9^{c}$ & $5.0^{e}$ & $4.7^{c}$ & $6.1^{d}$ & $0.2^{d}$ & $2.4^{c}$ & $0.2^{a b c}$ & $0.49^{c}$ & 33.3 \\
\hline Media & 2.1 & 6.6 & 6.1 & 7.6 & 0.35 & 3.1 & 0.19 & 0.62 & \\
\hline CV \% & 79.6 & 12.7 & 22.6 & 9.8 & 35.7 & 9.4 & 11.3 & 10.9 & \\
\hline
\end{tabular}

$\mathrm{cm}), \mathrm{CC}+\mathrm{G}+\mathrm{EO}+$ Suelo $(7.9 \mathrm{~cm})$ y $\mathrm{CC}+\mathrm{G}$ + Suelo $(6.3 \mathrm{~cm})$, mientras que los menores valores se tuvieron en el testigo $(4.7 \mathrm{~cm}), \mathrm{RV}+$ suelo y CC $+\mathrm{G}+\mathrm{B}+$ Suelo $(5.0 \mathrm{~cm})$. Los tratamientos que tuvieron el mayor número de hojas fueron $\mathrm{CC}+\mathrm{G}$ + EO (8.7), B + G + RV + Suelo (8.3) y la mayor biomasa la tuvo el tratamiento $\mathrm{CC}+\mathrm{G}+\mathrm{EO}+$ Suelo (0.5 g).

En la Tabla 5 se muestra el contenido de $\mathrm{N}$, $\mathrm{P}$ y K foliar, presentando el mayor contenido de $\mathrm{N}$ el tratamiento $B+G+R V+$ suelo $(3.9 \%)$, seguido por $\mathrm{B}+$ suelo $(3.4 \%)$, mientras que el menor contenido de $\mathrm{N}$ lo tuvo el testigo (2.4\%). El mayor contenido de $\mathrm{P}$ y $\mathrm{K}$ lo tuvieron los tratamientos $\mathrm{CC}+\mathrm{G}+\mathrm{RV}+$ suelo $(0.3 \%)$ y $\mathrm{CC}+\mathrm{G}+$ suelo $(0.74 \%)$ y $C C+G+E O+$ suelo $(0.72 \%)$.

\section{DISCUSIÓN}

\section{Desarrollo de la lombriz}

La sobrevivencia de las lombrices en los sustratos es un buen indicador de la calidad y estabilidad. Al respecto Evans y Percy (2014) señalan que los residuos orgánicos pueden contener agentes tóxicos cuando no se alcanza la estabilidad después del pre-composteo, lo que podría explicar la elevada tasa de mortalidad en el tratamiento EO + G. Al respecto Mupondi et al. (2011) indican que los tiempos de pre-composteo para la asimilación del sustrato por la lombriz dependen del origen de los materiales, pero Ndegwa y Thompson (2001) indican que con 30 días es suficiente para estabilizar la materia orgánica. La nula mortalidad en los tratamientos CC, CC + G + B y CC + G + RV se puede explicar por sus altos porcentajes de materia orgánica, lo que indica una alta disponibilidad de alimento y buena relación $\mathrm{C} / \mathrm{N}$ para el crecimiento de la lombriz (López-Méndez et al. 2013). Las diferencias en la producción de cocones se relacionan con el tipo de alimento, lo que influye en el desarrollo y la reproducción de la lombriz (Manivannan et al. 2009). En el tratamiento $E O+G$ no se produjeron cocones, posiblemente se deba a que las lombrices utilizaron la energía del alimento para la producción de biomasa, y no para la formación de cocones como lo indican Garg et al. (2005). Además, el exceso de carga proteica en la mezcla EO $+G$, puede causar cambios en el comportamiento de la lombriz.

El mayor número de lombrices juveniles se tuvo en los tratamientos $100 \%$ B y $100 \%$ RV, los cuales tuvieron altas relaciones $C / N$, lo que coincide con Aira et al. (2006). El tratamiento 50\% EO + $50 \%$ G tuvo la mayor biomasa promedio de lombriz (0.43 g), lo que indica una buena asimilación del sustrato, al respecto Senarathne y Llangamudali (2015) encontraron que los mayores pesos de lombrices se tuvieron cuando se alimentaron con mezclas de estiércol de ovino y G. sepium. Sobre lo mismo Garg et al. (2005) reportan que el 
incremento de la biomasa de la lombriz depende de la calidad del sustrato y de la competencia por el alimento. Las menores longitudes y biomasas de lombrices del tratamiento con cascarilla de cacao pueden deberse a las altas relaciones C/N (24 a 38 ), que indican una lenta asimilación y degradación de la materia orgánica.

\section{Características químicas de las vermicom- postas}

Los contenidos de $\mathrm{N}$ total de las vermicompostas dependen del nitrógeno inicial de los sustratos, del grado de descomposición de la materia orgánica (Garg et al. 2006) y de la mineralización (Abu et al. 2014). El pH alcalino de las vermicompostas puede atribuirse a la descomposición inicial de la materia orgánica, la formación de iones amonio, y a la generación de compuestos orgánicos de reacción alcalina (Shrimal y Khwairakpam 2010). La diferencia en los porcentajes de carbono puede estar relacionado con el origen del sustrato y sus combinaciones. Los mayores valores de $\mathrm{C}$ de la cascarilla de cacao y sus combinaciones, coinciden con los valores reportados por Sánchez et al. (2007). Mientras que los bajos valores de $C$ en el bocashí pueden deberse a los contenidos de azucares y levaduras que aceleran la proliferación de microorganismos e insectos que degradan la materia orgánica durante la fermentación (Boechat et al. 2013).

La relación $\mathrm{C} / \mathrm{N}$ refleja el grado de mineralización y estabilización de la materia orgánica durante el proceso de vermicomposteo (Suthar 2009). Los tratamientos con cascarilla de cacao tuvieron relaciones $\mathrm{C} / \mathrm{N}$ entre 24 y 38 , valores que en general son altos y se consideran adecuados para el desarrollo de microorganismos (MoralesMunguía et al., 2009). Al respecto Sánchez et al. (2007) indican que la relación $\mathrm{C} / \mathrm{N}$ es el factor más importante para la actividad biológica que ocurre durante la degradación de la materia orgánica, debido a que los microorganismos requieren carbono y nitrógeno como fuente de energía para crecer y multiplicarse. Las relaciones $\mathrm{C} / \mathrm{N}$ de los diferentes tratamientos indican la continua mineralización de los sustratos durante el proceso de vermicompostaje. En todos los sustratos la relación $\mathrm{C} / \mathrm{N}$ varió entre 23 y 38 , lo que indica aumentos en el contenido de nitrógeno. Al respecto, Yadav y Garg (2009) indica que una disminución en esta relación implica un aumento en el grado de humificación de la materia orgánica. Los contenidos de fósforo pueden explicarse por la composición del sustrato original, los lentos incrementos de fósforo observados reflejan una la lenta mineralización y movilización.

Los mayores valores de $\mathrm{CIC}$ se tuvieron en los tratamientos con CC, CC + G y CC + G + EO, aportando cationes que aumentan los contenidos de la fracción coloidal de los sustratos orgánicos (Trinidad-Santos 1987). En los tratamientos $100 \%$ CC, $50 \%$ CC $+50 \%$ G, y $50 \%$ CC $+25 \%$ G $+25 \%$ EO se encontraron altos valores de materia orgánica, lo que incrementó la $\mathrm{CIC}$, que es un excelente indicador para estimar el estado físico-químico de los sustratos orgánicos. Con respecto a los contenidos de $\mathrm{K}$ se obtuvo un comportamiento semejante a la $\mathrm{CIC}$, los diferentes valores pueden atribuirse al tipo de material utilizado, las combinaciones y la asimilación de este nutriente por parte de las lombrices durante el vermicompostaje. Debido a que las glándulas calcíferas en la mayoría de las lombrices son responsables de la liberación de carbonatos de calcio durante la ingestión de materia orgánica (Yan et al. 2013).

Valores bajos de ácidos húmicos se tuvieron en los tratamientos que contenían cascarilla de cacao, lo que difiere de lo reportado por Sánchez et al. (2007), quienes encontraron altos valores con la cacarilla de cacao. Al respecto se menciona que las cantidades de ácidos húmicos pueden atribuirse a la calidad de los sustratos (Asing et al. 2009).

\section{Germinación del chile habanero}

Los tratamientos con vermicompostas tuvieron los mayores porcentajes de germinación con respecto al téstigo, lo que coincide con Warman y Anglopez (2010). Se encontró que las vermicompostas con bocashí, tuvieron los mayores índices de germinación y crecimiento de las plántulas, al 


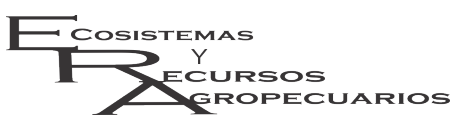

respecto Boechat et al. (2013) y Huerta et al. (2010) reportan que las vermicompostas con cascarilla de cacao tienen buenas respuestas en la germinación y crecimiento del chile. Por otro lado, las vermicompostas a base estiércol de ovino combinado con $G$. sepium (1:1) tuvieron efectos inhibidores en la germinación del Chile habanero, lo que se puede deber al alto contenidos de $\mathrm{N}$, que promueve la formación de sustancias tóxicas, que afectan de forma negativa la germinación, el desarrollo del sistema radical y el crecimiento de las plántulas de chile habanero.

\section{CONCLUSIONES}

Los tratamientos con residuos vegetales, bocashí y sus combinaciones tuvieron los mayores valores de biomasa, población, longitud, número de cocones y lombrices juveniles. El estiércol de ovino favorece la obtención de altos valores de biomasa y longitud de lombrices, mientras que los tratamientos con cascarilla de cacao mejoraron la producción de cocones al combinarse con G. sepium, residuos vegetales y bocashí. Altos porcentajes de nitrógeno y fósforo se obtienen al incluir estiércol de ovino y la combinación de estiércol de ovino $+G$. sepium. El uso del bocashí y su combinación con $G$. sepium, favoreció la germinación del chile habanero.

\section{LITERATURA CITADA}

Abu A, Nurul S, Mohd S, Zatina N, Abdullah N (2014) Vermicomposting of vegetable waste amended with different sources of agro-industrial by-product using Lumbricus rubellus. Polish Journal of Environmental Studies 23: 1491-1498.

Aira M, Monroy F, Domínguez J (2006) C to N ratio strongly affects population structure of Eisenia fetida in vermicomposting systems. European Journal of Soil Biology 42: S127- S131.

Asing J, Wong NC, Lau S (2009) Optimization of extraction method and characterization of humic acid derived from coals and composts. Journal of Tropical Agriculture and Food Science 37: 211-223.

Atiyeh RM, Lee S, Edwards CA, Arancon NQ, Metzger JD (2002) The influence of humic acids derived from earthworm-processed organic wastes on plant growth. Bioresource Technology 84:7-14.

Barreto CZA, López AJE, Radillo FF, Farías LJ, Bazán TM (2008) Uso de vermicomposta en suelo salino con plantas de Chile serrano (Capsicum annuum L.) en invernadero. Revista Institucional Universidad de Colima 25: 1-5

Batham M, Arya R, Tiwari A (2014) Time efficient co-composting of water hyacinth and industrial wastes by microbial degradation and subsequent vermicomposting. Journal of Bioremediation \& Biodegradation 5: 222.

Bazrafshan E, Zarei A, Kord Mostafapour F, Poormollae N, Mahmoodi S, Zazouli MA (2016) Maturity and stability evaluation of composted municipal solid wastes. Health Scope 5: e33202.

Bhat SA, Singh J, Vig AP (2015) Potential utilization of bagasse as feed material for earthworm Eisenia fetida and production of vermicompost. Springerplus 4: 11.

Boechat C, Gonzaga JA, Aguiar AM (2013) Net mineralization nitrogen and soil chemical changes with application of organic wastes with 'Fermented bokashi compost'. Acta Scientiarum Agronomy 35: 257-264.

Evans KJ, Percy AK (2014) Integrating compost teas in the management of fruit and foliar diseases for sustainable crop yield and quality. In: Maheshwari DK (ed.). Composting for sustainable agriculture. Springer. Switzerland. pp: 173-198. 
Garg V, Chand S, Chhillard A, Yadav A (2005) Growth and reproduction of Eisenia foetida in various animal wastes during vermicomposting. Applied Ecology and Environmental Research 3: 51-59.

Garg P, Gupta A, Satya S (2006) Vermicomposting of different types of waste using Eisenia foetida: A comparative study. Bioresource Technology 97: 391-395.

Huerta E, Vidal O, Jarquin A, Geissen V, Gomez R (2010) Effect of vermicompost on the growth and production of amashito pepper, interactions with earthworms and rhizobacteria. Compost Science \& Utilization 18: 282-288.

López-Méndez C, Ruelas-Ayala RD, Sañudo-Torres RR, Armenta-López C, Félix-Herrán JA (2013) Influencia de diferentes sustratos orgánicos en la lombriz roja californiana (Eisenia foetida). Tecnociencia Chihuahua 7: 81-87.

Majlessi M, Eslami A, Najafi S, Mirshafieean S, Babaii S (2012) Vermicomposting of food waste: assessing the stability and maturity. Iranian Journal of Environmental Health Sciences \& Engineering 9: 25.

Manivannan S, Balamurugan M, Parthasarathi K, Gunasekharan G, Ranganathan R (2009) Effect of vermicompost on soil fertility and crop productivity-beans (Phaseolus vulgaris). Journal of Environmental Biology 30: 275-28.

McGinnis MS, Warren SL, Bilderback TE (2009) Replacing conventional nursery crop nutrient inputs with vermicompost for container production of Hibiscus moscheutos L. "Luna Blush". HortScience 44: 16981703.

Morales-Munguía JC, Fernández-Ramírez, A, Montiel-Cota BC, Peralta-Beltrán (2009) Evaluación de sustratos orgánicos en la producción de lombricomposta y el desarrollo de lombriz (Eisenia foetida). BIOtecnia 11: 19-26.

Moreno-Reséndez A, Zarate T, Valdés PMT (2005) Desarrollo del tomate en sustrato de vermicomposta/arena bajo condiciones de invernadero. Agricultura Técnica 65:27-34.

Mupondi LT, Mnkeni PN, Muchaonyerwa P (2011) Effects of a precomposting step on the vermicomposting of dairy manure waste paper mixtures. Waste Management \& Research 29: 219-228.

Nelson DW, Somers LE (1996) Total carbon, organic carbon and organic matter. In: Spark DL (ed). Methods of soil analysis. Part 3 Chemical methods. Soils Science Society of American Books Series no. 5. USA. pp: 961-1010.

Ndegwa PM, Thompson SA (2001) Integrating composting and vermicomposting in the treatment and bioconversion of biosolids. Bioresource Technology 76: 107-112.

Nieto-Garibay A, Murillo AB, Troyo DE, Larrinaga MJA, García HJL (2002) El uso de composta como alternativa ecológica para la producción sostenible de Chile (Capsicum chinense Jacq.) en zonas áridas interciencia 27: 417-421.

Palma-López DJ, Cisneros-Domínguez J, Moreno CE, Rincón-Ramírez JA (2007) Suelos de Tabasco: Su uso y manejo sustentable. Colegio de Postgraduados, ISPROTAB, Fundación Produce Tabasco AC, Villahermosa, Tabasco, México, pp. 93.

Ruiz-Bello R, Nava-Tablada ML, Landeros-Sánchez C, Díaz-Padilla G (2016) Potencial productivo y limitantes para el cultivo de chile habanero (Capsicum chinense. Jacq.) en el estado de Veracruz, México. Revista Internacional de Desarrollo Regional Sustentable 1: 1-11.

Ramos D, Terry E (2014) Generalidades de los abonos orgánicos: Importanica del bocashi como alternativa nutricional para suelos y plantas. Cultivos Tropicales 35: 52-59. 
Rodríguez-Dimas DN, Cano-Ríos P, Favela-Chávez E, Figueroa-Viramontes U, Paul-Álvarez V, Palomo-Gil A, et al. (2007) Vermicomposta como alternativa orgánica en la producción de tomate en invernadero. Revista Chapingo Serie Horticultura 13: 185-192.

Rodríguez DN, Cano P, Figueroa VU, Palomo GA, Favela Che, Álvarez RVP, et al. (2008) Producción de tomate en invernadero con humus de lombriz como sustrato. Revista Fitotecnia Mexicana 31: 265-272.

Ruíz-Bello R, Nava-Tablada ME, Landeros-Sánchez C, Días-Padilla G (2016) Potencial productivo y limitantes para el cultivo de Chile habanero (Capsicum chinense Jacq.) en el estado de Veracruz, México. Revista Internacional de Desarrollo Regional Sustentable 1: 1-11.

Sánchez R, Ordaz VM, Valdes SB, Palma-López DJ, Bolón J., (2007) Chemical characteristics of several vermicomposts in México. Compost Science \& Utilization 15: 47-52.

SEMARNAT (2000) Norma Oficial Mexicana NOM-021 (AS-02, AS-12, AS-25) Que establece las especificaciones de fertilidad, salinidad y clasificación de suelos, estudio, muestreo y análisis. Secretaría de Medio Ambiente y Recursos Naturales, publicado en el Diario Oficial de la Federación el 23 de abril de 2003.

Senarathne SH, Llangamudali IMPS (2015) Utilization of different plant species available in coconut plantation to produce nutrient rich vermicompost. The Journal of the Coconut Research Institute of Sri Lanka 21: 33-42.

Shrimal S, Khwairakpam M (2010) Effect of C/N ratio on vermicomposting of vegetable waste. Dinamic Soil, Dinamic Plant 4: 123-126.

Suthar S (2009) Vermicomposting of vegetable market solid waste using Eisenia fetida: Impact of bulking materialon earthworm growth and decomposition rate. Ecological Engineering 35: 914-920.

Tamayo J, Martínez E, Monforte G, Munguía A, Ruíz A (2014) La Agroecología como propuesta de modelo de producción aplicado al cultivo del Chile habanero en Peto Yucatán. Revista Mexicana de Agronegocios 35: 969-978.

Trinidad-Santos A (1987) El uso de los abonos orgánicos en la producción agrícola. Serie Cuadernos de Edafología número 10. Centro de edafología, Colegio de Postgraduado, Chapingo, México. 7p.

Warman PR, Anglopez MJ (2010) Vermicompost derived from different feedstocks as a plant growth medium. Bioresource Technology 101: 4479-4483.

Yadav A, Garg VK (2009) Feasibility of nutrient recovery from industrial sludge by vermicomposting technology. Journal Hazardous Materials 168: 262-268.

Yan YW, Azwady AAN, Shamsuddin ZH, Muskhazli M, Aziz SA, Teng SK (2013) Comparison of plant nutrient contents in vermicompost from selected plant residues. African Journal of Biotechnology 17: 2207-2214. 